LetTers to the Editor

\title{
The Effect of Termination of Membrane Phospholipid Synthesis on Cell-dependent Events in Caulobacter
}

\begin{abstract}
Membrane phospholipid synthesis in Caulobacter crescentus has been shown to be related to the expression of specific cell cycle events. DNA synthesis was immediately inhibited if phospholipid synthesis was terminated either by glycerol starvation of a glycerol auxotroph or by treatment of mutant and wildtype cultures with cerulenin. Termination of phospholipid synthesis, by either method, resulted in the inhibition of stalk elongation, flagellum biogenesis and cell division. The inability to form a stalk appears to be directly due to the cessation of phospholipid synthesis, whereas the inhibition of flagella formation and cell division is likely a result of the secondary effect on DNA replication. Two cell cycle events, the ejection of the flagellum and stalk initiation, were shown to be independent of phospholipid synthesis and DNA replication.
\end{abstract}

Many cellular changes which occur during the cell cycle of Caulobacter crescentus involve the cell membrane, including cell division and the formation of surface organelles such as stalk, flagella and pili. These changes at the cell surface occur at defined sites and this spatial restriction seems to be rigorously controlled (Newton et al., 1975; Shapiro, 1976; Agabian et al., 1979). It is therefore likely that membrane biogenesis is an integral component of the regulatory network that controls both the temporal and spatial expression of surface morphogenesis. We have addressed the question of whether a specific relationship exists between membrane synthesis and the expression of cell cycle events by analyzing membrane phospholipid synthesis in C. crescentus.

The composition of the membrane phospholipids in $C$. crescentus is relatively simple; $87 \%$ of the phospholipids are phosphatidylglycerol and cardiolipin (Contreras et al., 1978; Jones \& Smith, 1979). We have shown that the pattern of phospholipid synthesis varies during the cell cycle: the turnover rate of phosphatidylglycerol precursors is markedly different in swarmer and stalked cells (Mansour et al., 1980). Furthermore, three additional minor phospholipids can be detected in swarmer cells. In order to facilitate the analysis of phospholipid biosynthesis and its relationship to the expression of cell cycle events, we have isolated mutants of $C$. crescentus altered in biosynthetic sn-glycerol-3-phosphate dehydrogenase activity which are dependent on exogenous glycerol for phospholipid biosynthesis (Contreras et al., 1979). In this report we manipulate phospholipid synthesis both by glycerol deprivation of the glycerol auxotroph and by treatment of mutant and wild-type cells with the antibiotic cerulenin. Cerulenin has been shown to inhibit phospholipid syuthesis by specifically blocking $\beta$-ketoacylthioester synthetase activity (D'Agnolo et al., 1973; Goldberg et al., 1973). Using these techniques to inhibit phospholipid synthesis we show here that DNA synthesis in Caulobacter is dependent on net phospholipid synthesis. We also observed that specific cell cycle events were blocked when net phospholipid synthesis was terminated. Since it has been demonstrated 
that DNA synthesis is necessary for flagellum biogenesis and the initiation of the cell division pathway in $C$. crescentus (Degnan \& Newton, 1972b; Newton et al., 1975; Osley et al., 1977), it is likely that the inhibition of DNA synthesis caused by the termination of phospholipid synthesis is reponsible for the subsequent inhibition of certain cell cycle-dependent events. Two cell cycle events, the ejection of the flagellum and the initiation of stalk formation, however, appear to be independent of phospholipid synthesis.

(a) The effect of the inhibition of phospholipid synthesis on

\section{$D N A$ and RNA synthesis}

Phospholipid synthesis in $C$. crescentus was inhibited by two independent means; by glycerol starvation of a glycerol auxotroph and by treatment of cell cultures with the antibiotic cerulenin. The glycerol auxotroph AE5168 was grown either in the presence or in the absence of glycerol (Fig. 1(a)). The requirement for glycerol by strain AE5168 was previously shown to be due to its very low levels of the biosynthetic sn-glycerol-3-phosphate dehydrogenase (Contreras et al,, 1979). The net synthesis of phospholipids in this mutant ceased immediately after glycerol deprivation (Fig. 1; Contreras et al., 1979). When the glycerol auxotroph was deprived of glycerol and phospholipid synthesis terminated, net RNA synthesis continued for at least the equivalent of one generation $(240 \mathrm{~min})$. The net synthesis of DNA, however, ceased as early as one-eighth of a generation time after glycerol deprivation, suggesting that inhibition of DNA synthesis occurred before a round of DNA replication was completed (Fig. 1(a)). In revertant strain AE5171, net DNA synthesis in the absence of glycerol was indistinguishable from that observed in the mutant AE5168 grown in the presence of glycerol.

DNA and RNA synthesis were then measured in cerulenin-treated cultures in order to determine if the inhibition of DNA replication observed upon glycerol starvation of mutant AE5168 was a response to the cessation of phospholipid synthesis or reflected some other alteration in a metabolic function specific for this mutant. The antibiotic cerulenin has been shown to inhibit phospholipid synthesis by blocking fatty acid synthesis (Omura, 1976). Figure 1(b) shows that upon inhibition of net phospholipid synthesis with cerulenin, net DNA synthesis was terminated, whereas RNA synthesis continued for 80 minutes before stopping. Identical results were obtained both with strain AE5168 grown in the presence of glycerol and with the parent strain AE5091. Similar studies in other bacteria have shown that following the addition of cerulenin and the consequent inhibition of phospholipid synthesis, the rate of RNA synthesis declined, but the rate of DNA synthesis remained unaffected (Wille et al., 1975) or decreased a maximum of $25 \%$ (Goldberg et al., 1973).

Since the inhibition of phospholipid synthesis in $C$. crescentus appeared to result in the cessation of DNA replication, we analyzed the ability of $C$. crescentus to synthesize phospholipids under conditions in which DNA synthesis was specifically inhibited. Accordingly, net phospholipid synthesis was measured in the temperaturesensitive DNA chain elongation mutant PC1042 (Osley \& Newton, 1977) grown at the permissive $\left(30^{\circ} \mathrm{C}\right)$ and restrictive $\left(37^{\circ} \mathrm{C}\right)$ temperature; phospholipid synthesis proceeded normally at both $30^{\circ} \mathrm{C}$ and $37^{\circ} \mathrm{C}$. These results indicate, therefore, that 


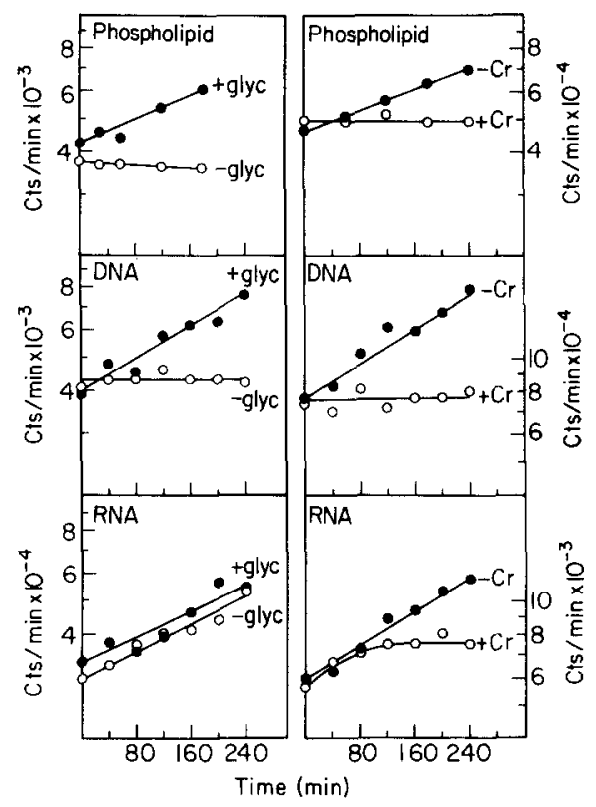

(a)

(b)

FIG. 1. Net synthesis of phospholipids, DNA and RNA in C. crescentus AE5168. Cultures were grown in glycerol $(0.2 \%, \mathrm{v} / \mathrm{v})$-supplemented M2 minimal media (Johnson \& Ely, 1977) in the presence of ${ }^{32} P_{1}$ for 5 generations. The final specific activity of ${ }^{32} \mathrm{P}$ in the growth medium was 0.5 $\mathrm{mCi} / \mathrm{mmol}$. The generation time in these experiments was $240 \mathrm{~min}$. The cells were sedimented at $10,000 \mathrm{~g}$ for $5 \mathrm{~min}$, washed and resuspended in M2-glucose medium at the same ${ }^{32} \mathrm{P}$ specific activity (a) in the presence and absence of glycerol, and (b) in the presence of glycerol but with and without cerulenin $(20 \mu \mathrm{g} / \mathrm{ml})$. Portions $(10 \mathrm{ml})$ of each culture were withdrawn at various times, filtered through $0.45 \mu \mathrm{m}$ Millipore filters and washed with $\mathrm{M2}$ medium. The cells were suspended in M2 medium and the phospholipids were extracted with methanol/chloroform as previously described (Contreras et al., 1978). The number of counts in $0.1 \mathrm{ml}$ portions of the chloroform extracts were measured in a liquid scintillation counter. Portions $(0.1 \mathrm{ml})$ of the same culture were removed at various times and precipitated with $10 \%$ trichloroacetic acid in order to measure RNA and DNA synthesis. The samples were precipitated with trichloroacetic acid, filtered, washed and counted as described by Degnan \& Newton $(1972 a, b)$. Simultaneously, portions $(0 \cdot 1 \mathrm{ml})$ were removed and precipitated with $10 \%$ trichloroacetic acid. The precipitates were collected by centrifugation, resuspended in $1 \mathrm{M} \cdot \mathrm{KOH}$ and incubated for $16 \mathrm{~h}$ at $37^{\circ} \mathrm{C}$. The samples were neutralized, reprecipitated with cold $10 \%$ trichloroacetic acid, filtered, washed with $5 \%$ trichloroacetic acid and counted. The counts incorporated into DNA were obtained by subtracting alkali-labile counts from the total counts incorporated.

phospholipid synthesis is not dependent on DNA replication, but that phospholipid synthesis is necessary for DNA replication.

\section{(b) Effect of the inhibition of phospholipid synthesis on the progress}

$$
\text { through the cell cycle }
$$

A striking consequence of the termination of phospholipid synthesis in either the glycerol auxotroph or in cerulenin-treated cultures was the early loss of motility. We therefore investigated this reponse both with respect to the distribution of cell types and the synthesis of specific temporally regulated proteins. 
In order to determine if log phase cultures were blocked at a specific stage in the cell cycle upon the termination of phospholipid synthesis, the glycerol auxotroph AE5168 was grown in the presence or absence of glycerol and examined in the electron microscope. The relative proportion of the various cell types, shown schematically in Figure 2, was determined by analyzing micrographs of severl hundred cells grown in the presence or absence of glycerol (Table 1). A revertant, AE5171 (Contreras et al., 1979) grown in the absence of glycerol, was also analyzed in this manner. The proportion of the cell types in cultures of the mutant AE5168 grown in the presence of glycerol or of the revertant AE5171 grown in the absence of glycerol agreed with the normal distribution of $C$. crescentus cells recently determined by R. Tax (Osley \& Newton, 1978). However, small cells with short, aberrant stalks comprised approximately $25 \%$ of the total nnutant cell population under conditions of starvation

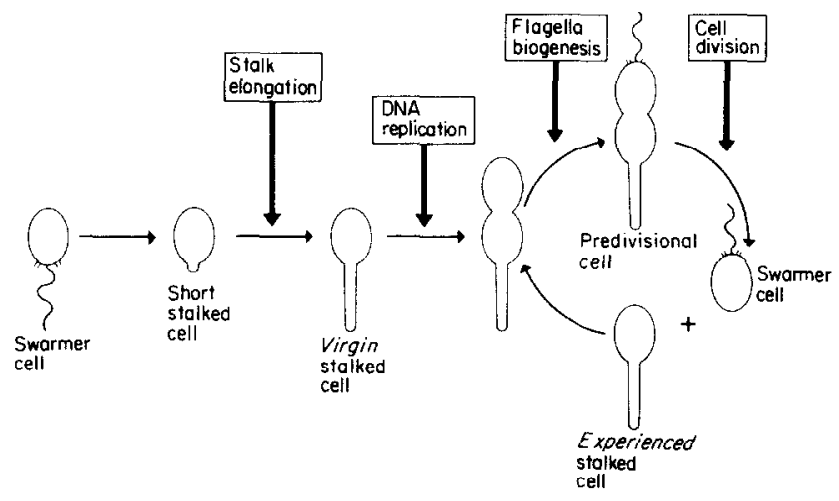

FIG. 2. Schematic representation of $C$. crescentus life cycle. Arrows indicate cell cycle events which are blocked by the cessation of phospholipid synthesis.

\section{Table I}

Distribution of mutant and revertant cell types grown in the presence and absence of glycerol

\begin{tabular}{|c|c|c|c|c|c|c|}
\hline \multirow{3}{*}{ Cell type } & \multicolumn{4}{|c|}{ Mutant AE5168 } & \multirow{2}{*}{\multicolumn{2}{|c|}{$\begin{array}{c}\text { Revertant AE5171 } \\
\text { - glycerol }\end{array}$}} \\
\hline & \multicolumn{2}{|c|}{+ glycerol } & \multicolumn{2}{|c|}{ - glycerol } & & \\
\hline & Cell no. & $\%$ & Cell no. & $\%$ & Cell no. & $\%$ \\
\hline Stalked cell & 114 & $53 \cdot 2$ & 154 & $38 \cdot 4$ & 120 & $45 \cdot 6$ \\
\hline Short stalked cell & 11 & $5 \cdot 0$ & 103 & $25 \cdot 7$ & 11 & $4 \cdot 2$ \\
\hline Swarmer cell & 51 & $\overline{23 \cdot 8}$ & 39 & $\overline{9 \cdot 7}$ & 88 & $\overline{33 \cdot 5}$ \\
\hline Predivisional cell & 36 & $\overline{16 \cdot 8}$ & 80 & $2 \overline{0 \cdot 0}$ & 43 & $\overline{16 \cdot 3}$ \\
\hline Aberrant cell & 2 & 0.9 & 25 & $6 \cdot 2$ & 1 & 0.4 \\
\hline
\end{tabular}

Cultures of mutant AE5168 and the revertant strain AE517l were incubated in M2-glucose minimal medium in the presence or absence of glycerol ( $1 \mathrm{~mm}$ ), as indicated, for $240 \mathrm{~min}$ at $30^{\circ} \mathrm{C}$. Electron micrographs of negatively stained culture samples were prepared and cell types scored. In mutant cultures incubated in the absence of glycerol cells were scored as "swarmers" if they were small and lacked any visible stalk. 
for glycerol (Table 1). The observation that the proportion of cells with short stalks was equivalent to the proportion of the total cell population normally occupied by swarmer cells indicates that upon termination of phospholipid synthesis the existing swarmer cells shed their flagella, initiated stalk formation, but stalk elongation failed to occur. Predivisional cells with short stalks were never observed, suggesting that cells which reached the "short stalk" stage (see Fig. 2) did not proceed any further in the cell cycle. To test this hypothesis, swarmer cells were isolated (Lagenaur et al., 1977) from log phase cultures of AE5168 and then synchronous growth in the presence and absence of glycerol was monitored in the electron microscope (Fig. 3). Both stalked cells and predivisional cells were observed in the glycerol-supplemented culture. In the absence of glycerol, however, $98 \%$ of the swarmer cells had shed their flagella and accumulated at the short-stalked cell stage, confirming that glycerol deprivation allowed stalk initiation but prevented stalk elongation. We have recently observed that continued phospholipid synthesis, but not DNA synthesis, is required for stalk elongation.

Viable count measurements and cell number determined in a Coulter counter (Osley \& Mansour, unpublished results) indicated that another stage in the cell cycle blocked by both glycerol deprivation and treatment with cerulenin was cell division. Since it was observed that the inhibition of phospholipid synthesis resulted in the termination of net DNA synthesis (see Fig. 1), the concomitant inhibition of cell division is in keeping with the dependency of cell division on DNA replication demonstrated by Degnan \& Newton $(1972 b)$.

Since it has been demonstrated that flagellin synthesis is dependent on DN $\Lambda$ replication in $C$. crescentus (Osley et al., 1977), we questioned whether the loss of flagella following the cessation of phospholipid synthesis was due to a block in the synthesis of flagellum protein components, or to a block in the assembly of the flagellar structure. The inability to form the flagella structure was not due to a generalized effect on protein synthesis, since we have shown that protein synthesis continues for at least four hours after the termination of phospholipid synthesis (Contreras et al., 1979).

It has been demonstrated that the components of the flagellar structure, flagellin A, flagellin B, and hook protein, are synthesized just prior to their assembly on the predivisional cell (Shapiro \& Maizel, 1973; Lagenaur \& Agabian, 1978; Agabian et al., 1979; Osley \& Newton, 1977; Osley et al., 1977). The synthesis of these components was analyzed during the inhibition of phospholipid synthesis by preparing immunoprecipitates of labeled cell extracts with specific antibody at various times after glycerol deprivation of the mutant AE5168. Anti-flagellin antibody and antihook protein antibody were used in two separate experiments and it was observed that the synthesis of flagellin $A$, flagellin $B$, and the hook protein, respectively, was inhibited as early as one hour after glycerol deprivation. Under these same conditions of glycerol deprivation, mutant AE5168 was observed to continue synthesizing other proteins. For example, autoradiograms of proteins precipitated with anti-RNA polymerase antibody and separated by gel electrophoresis showed that the $\beta \beta^{\prime} \sigma$ and $\alpha$ subunits continued to be synthesized four hours after removal of glycerol from mutant cultures. It appears therefore that the absence of flagella observed in cultures deprived of glycerol was due to the specific inhibition of synthesis of the 

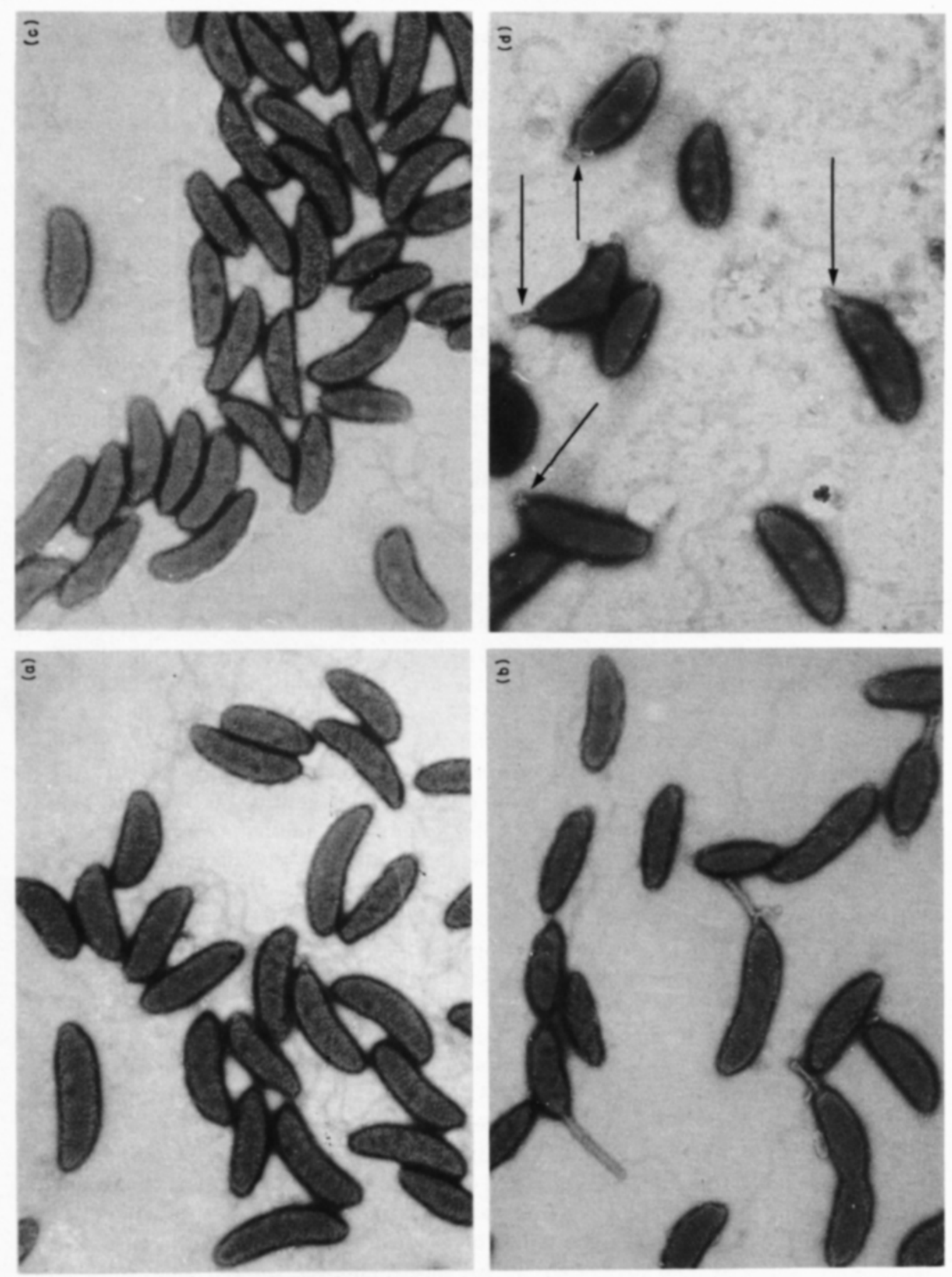
flagellar protein components and is likely due to the inhibition of DNA replication soon after the removal of glycerol.

We have shown in this report that upon termination of phospholipid synthesis in C. crescentus either by glycerol deprivation of a glycerol auxotroph or by treatment with the antibiotic cerulenin, an immediate effect is the inhibition of DNA synthesis, while RNA and protein synthesis continue. Measurement of net DNA synthesis after the termination of phospholipid synthesis in exponentially growing cultures showed that DNA chain elongation, rather than the initiation of DNA replication, was inhibited. Using Osley and Newton's temperature-sensitive DNA chain elongation mutant PC1042, we observed that the inhibition of DNA synthesis did not cause the termination of phospholipid synthesis. It therefore appears that while DNA synthesis is dependent on phospholipid synthesis the reverse is not true. The termination of phospholipid synthesis also resulted in the inhibition of stalk elongation, flagellum biogenesis and cell division. Although the inability to form a stalk under these conditions appears to be directly due to the inhibition of phospholipid synthesis, the inhibition of both flagellum formation and cell division are likely a result of the secondary termination of DNA replication.

The mechanism by which ongoing phospholipid synthesis could interact with the multiple factors involved in DNA initiation and elongation in Caulobacter is not known. Thilo \& Vielmetter (1976) have provided evidence that DNA replication in Escherichia coli is independent of membrane lipid composition as well as the extent of membrane fluidity. Although it appears likely that lipids are randomly inserted into the bacterial membrane and quickly diffuse (Thilo \& Vielmetter, 1976; Thilo \& Overath, 1976), biochemical parameters related to the synthesis of these cell components may in fact provide a means of "cogwheeling" the two phenomena. For example, a phospholipid precursor available only during active phospholipid synthesis may act as a cofactor for DNA elongation in $C$. crescentus. We have shown that although the overall phospholipid composition of the $C$. crescentus cell membrane remains essentially unchanged after the inhibition of phospholipid synthesis (Contreras et al., 1979), the pattern of precursor synthesis and turnover is markedly different in swarmer cells and stalked cells (Mansour et al., 1980). This observation is particularly pertinent, since DNA replication has been shown to be specifically repressed in $C$. crescentus swarmer cells but not stalked cells (Degnan \& Newton, 1972a). Experiments are currently in progress to discriminate between a diffusable cofactor and a physical interaction between membrane and replicating chromosome by determining whether the termination of phospholipid synthesis affects the replication of a foreign phage or plasmid DNA under conditions where chromosomal replication is shown to be inhibited.

The inhibition of DNA synthesis in $C$. crescentus upon the cessation of membrane phospholipid synthesis is so rapid as to suggest that it reflects an early step in this pathway. One candidate for this altered early step might be damage to a replication

Fig. 3. Flectron micrograph of C. crescentus AE5 168 swarmer cells grown in the presence (a) and (b) and absence (c) and (d) of glycerol $(0.2 \% \mathrm{v} / \mathrm{v})$. (a) and (c) show cell population at 0 time, and (b) and (d) show cell population at $200 \mathrm{~min}$. Arrows show very short stalks. Magnification, $10,400 \times$. Bacterial preparations were negatively stained with $1 \%(\mathrm{w} / \mathrm{v})$ phosphotungstic acid using the procedure described previously (Shapiro \& Maizel, 1973). 
complex which perhaps requires ongoing membrane synthesis for its integrity. A physical interaction between the membrane and the replicating chromosome, although difficult either to define or demonstrate, is particularly attractive as a mechanism to regulate the rigorous polarity exhibited by $C$. crescentus. The flagellum is assembled at the cell pole from protein components which are synthesized at specific times in the cell cycle and then sent through a specific site in the cell membrane. DNA replication has been shown to be required for flagellin synthesis (Osley et al., 1977). Perhaps the replicating chromosome, localized at the cell pole, signals flagellin synthesis at the time that the flagellin gene is replicated, and the localized product is then available at the correct site on the membrane for assembly into the flagellar structure. Recently, Evinger \& Agabian (1979) have demonstrated that the envelopeassociated nucleoids isolated from swarmer and stalked cells have very different sedimentation coefficients, perhaps indicating a relationship among differentially expressed proteins and a specific DNA-membrane complex.

This investigation was supported by grant no. GB42545X from the National Science Foundation (to L. S.), and grant no. GM 11301 from the National Institutes of Health (to L. S.), and grant no. GM 19629 from the National Institutes of Health to another author (S. H.), and by the Core Cancer Grant NIH/NCI P30CA13330-7. One of the authors (L. S.) is a recipient of a Hirschl Trust Award and S. H. is a recipient of a Research Career Development Award (GM-00024) from the National Institutes of Health. One author (R. B.) is a recipient of an American Cancer Society Junior Faculty Research Award.

We thank M. A. Osley and A. Newtom for the Ts DNA mutant and Jane Fant for her excellent assistance with the electron microscopy.

Departments of Molecular Biology and Genetics

Division of Biological Sciences

Albert Einstein College of Medicine

Bronx, New York 10461, U.S.A.

INES CONTRERAS

A. WEISSBORN

K. AMEMIYA

J. Mansour

S. HENRY

LUCILle Shapiro

Department of Microbiology

R. BENDER

University of Michigan

Ann Arbor, Michigan, U.S.A.

Received 19 September 1979, and in revised form 3 December 1979

\section{REFERENCES}

Agabian, N., Evinger, M. \& Parker, G. (1979). J. Cell. Biol. 81, 123-126.

Contreras, I., Shapiro, L. \& Henry, S. (1978). J. Bacteriol. 135, 1130-1136.

Contreras, I., Bender, R., Mansour, J., Henry, S. \& Shapiro, L. (1979). J. Bacteriol. $140,612-619$.

D'Agnolo, G. I., Rosenfeld, S., Awaya, J., Omura, S. \& Vagelos, P. R. (1973). Biochim.

Biophys. Acta, 326, 155-156.

Degnan, S. T. \& Newton, A. (1972a). J. Mol. Biol. 64, 671-680.

Degnan, S. T. \& Newton, A. (1972b). J. Bacteriol. 110, 852-856.

Evinger, M. \& Agabian, N. (1979). Proc. Nat. Acad. Sci., U.S.A. 76, 175-178.

Goldberg, I., Walker, J. R. \& Bloch, K. (1973). Antimicrob. Agents Chemo. 3, 549-554.

Johnson, R. C. \& Ely, B. (1977). Genetics, 86, 25-32. 
Jones, D. E. \& Smith, J. D. (1979). Canad. J. Biochem. 57, 424-428.

Lagenaur, C. \& Agabian, N. (1978). J. Bacteriol. 135, $1062-1069$.

Lagenaur, C., Farmer, S. \& Agabian, N. (1977). Virology, 77, 401-407.

Mansour, J., Henry, S. \& Shapiro, L. (1980). J. Bacteriol. In the press.

Newton, A., Osley, M. A. \& Terrana, B. (1975) In Microbiology-1975 (Schlessinger, D., ed.), American Society for Microbiology, Washington, D. C.

Omura, S. (1976). Bacteriol. Rev. 40, 681-697.

Osley, M. A. \& Newton, A. (1977) Proc. Nat. Acad. Sci., U.S.A. 74, 124-128.

Osley, M. A. \& Newton, A. (1978). J. Bacteriol. 135, $10-17$.

Osley, M. A., Sheffery, M. \& Newton, A. (1977). Cell, 12, 393-400.

Shapirn, I. (1976). Annu. Rev. Microbiol. 30, 377-407.

Shapiro, L. \& Maizel, J. V. (1973). J. Bacteriol. 113, 478-485.

Thilo, L. \& Overath, D. (1976). Biochemistry, 15, 328-334.

Thilo, L. \& Vielmetter, W. (1976). J. Bacteriol. 128, 130-143.

Wille, W., Eisenstadt, E. \& Willecke, K. (1975). Antimicrob. Agents Chemo. 8, 231-237. 